\title{
Salvage Surgery in Head and Neck Patients: Personal Experience and Case Report
}

\author{
Stefano Dallari
}

\begin{abstract}
Salvage surgery is always a challenging choice and must even more fulfil both oncologic requirements and quality-of-life issues. In head and neck patients, the most important goals are pain relief, preservation of breathing and deglutition, acceptable cosmetic results, and a sufficient quality of the residual life. When surgery is still considered an option, a safe, quick-recovering, and function-saving technique should be chosen. The author's experience and an illustrative case report are described.
\end{abstract}

Keywords: Head and neck surgery, Mandibular reconstruction, Oncological resection, Quality of life, Salvage surgery. International Journal of Head and Neck Surgery (2018): 10.5005/jp-journals-10001-1358

\section{INTRODUCTION}

A cancer regrowth in head and neck patients who already underwent a multimodality treatment is an even more difficult challenge to deal with. When considering such cases, the tumor board has to take into account several issues such as pain control, breathing and feeding necessities, cosmetic disfigurement, social environment, and quality of the residual life. If a surgical salvage is still considered feasible, it should be reasonably safe, require a short hospital stay, and guarantee an acceptable functional and cosmetic result. We adopted this philosophy in two recently treated patients, one-hereby reported-with a tumor regrowth after chemotherapy, transoral ablative surgery and radiotherapy for a T4 squamocellular carcinoma of the left tonsil, the other with a cancer recurrence at the tracheotomy site after a successful supracricoid laryngectomy for a persistent glottic cancer. ${ }^{1}$

\section{Case Description}

A 58-year-old man was referred to us (November 2012) for a T4, $\mathrm{N} 2, \mathrm{M} 0$ squamocellular carcinoma of the left tonsil. We consider the tumor initially unresectable with radicality expectations and planned a neo-adjuvant chemotherapy (platinum-FU for three sessions), after which we obtained a $85 \%$ reduction of T and a $50 \%$ reduction of $\mathrm{N}$. We discussed the case in our oncologic board and counseled the patient. The decision was to prefer an immediate surgery followed by radiotherapy. The patient underwent a transoral wide left tonsillectomy (frozen sections free from tumor) and a II-V modified radical neck dissection with preservation of the sternocleidomastoid muscle and spinal accessory nerve (February 2013).

The postoperative period was uneventful and the patient underwent a six-week radiation treatment ended in May 2013.

The follow up was negative for six months, when we detected a mass in the left level one (November 2013).

Fine needle aspiration cytology (FNAC) was positive for squamous cancer cells and we performed a bilateral submental and left submandibular dissection. The follow up remained negative until October 2014 when the left tonsillar region showed a de-epithelization, initially congruous with post-radiation sequelae.
Department of Otorhinolaryngology, Ospedale "A. Murri", Fermo, Italy Corresponding Author: Stefano Dallari, Department of Otorhinolaryngology, Ospedale "A. Murri", Fermo, Italy, Phone: +39333 1235287, e-mail: dallarinew@libero.it

How to cite this article: Dallari S. Salvage Surgery in Head and Neck Patients: Personal Experience and Case Report. Int J Head Neck Surg 2018;9(4):124-127.

Source of support: Nil

Conflict of interest: None

Fifteen days of antibiotic and antifungal therapy failed to improve the symptoms, with increasing local pain and initial trismus.

A biopsy was performed and squamous carcinoma cells mixed with a necrotic tissue were detected. The decision about what to do ranged from a palliative treatment to a salvage surgery. We were dealing with a young patient without other significant morbidities and a very painful situation.

We thoroughly counseled him and his family and proposed a transmandibular left amygdalo-glosso-palatine resection. Our first goal would be to relieve the pain and try to offer the patient the best quality of his residual life. Regarding the reconstruction, we focused our planning toward a relatively safe technique allowing the shortest hospital stay and an acceptable functional and cosmetic result. On December 23, 2014 the patient underwent the surgery.

We performed the tracheotomy under local anesthesia and then switched to general anesthesia and put the tube through the tracheotomy. Percutaneous endoscopic gastrostomy (PEG) was positioned, too. Left hemimandible was approached through the scar of the previous neck dissection and skeletonized from the notch to the mental foramen.

An L-angled titanium left mandible prosthesis was cut at a proper dimension and preplated. Then the mandibular angle was cut with an oscillating saw after controlling the amount of intraoral soft tissue to be excised. The 36 dental element was also extracted.

Once mobilized the bone of the mandibular angle, the tumor regrowth was approached intraorally and a wide excision with a 1.5-cm macroscopic free margin was carried out. On the perimeter of the excised area, several frozen sections were examined, all resulting free from the disease. 
The specimen was then detached in continuity with the underlying tissue and the mandibular bone. Further revision of the surgical amygdaloglosso-palatine ablation with accurate hemostasis was accomplished.

A classical left pectoralis major myocutaneous flap was then harvested, with a cutaneous skin paddle matching the intraoral defect. The paddle was transferred to obliterate the surgical defect and sutured with long-lasting resorbable 2-0 and 3-0 interrupted sutures in a water-tight manner. Once the oral defect was closed, the mandibular reconstruction took place. The previously prepared titanium plate was fixed with screws, achieving a perfect anatomic mandibular repositioning, with reconstitution of a normal dental occlusion.

Mandibular plating was reinforced with the remnant of the titanium prosthesis fixed anteriorly and superiorly in a triangular manner (Fig. 1). The muscular pedicle of the flap was then draped around the titanium plates and the skin was closed directly over it with a soft rubber drain. The postoperative outcome was better than expected with no complications. Immediately after recovering from the anesthesia, the patient referred the absence of pain and nothing but the standard pain control protocol was needed. The patient was discharged on the 12th postoperative day with the tracheotomy tube closed, feeding through the PEG and with a program of swallowing rehabilitation on an outpatient basis. Sufficient oral solid and fluid intake was reached four months after the operation (April 2015) and both the tracheal tube and the PEG were subsequently removed.

At four month control (April 2015), there was a good result with perfect flap integration and no sign of cutaneous sufferance in the zone of the titanium plaques reconstruction (Fig. 2). Good esthetic appearance and function were present and the patient was both satisfied and grateful. He went back to a substantially normal life and to his work (baker).

Six months after the operation (June 2015), a 2-cm node appeared in the retromandibular region. FNAC was positive for squamocellular cancer cells and a wide excision was performed under local anesthesia. The perinodular excised tissue was histologically free from the disease. The patient continued to lead his normal life and work (Fig. 3), and we planned a two-month-based follow-up. At the beginning of October 2015, the patient came to us complaining of a left perimandibular swelling and a slight pain. At the endoral examination, edema, de-epithelialisation, and initial fissuration of the cutaneous paddle were evident. Ultrasound examination showed a subcutaneous infiltration consistent with a malignant recurrence. Transoral biopsy confirmed the malignancy.

Palliative chemotherapy was then advised (carboplatin + taxol). After the first course, a complete remission of the cutaneous and deep lesions were detected endoscopically and at the ultrasound check, with no more swelling and pain. The patient well tolerated the treatment and he is doing his normal life with a program of further chemotherapy courses adjusted to the clinical conditions (Fig. 4).

\section{Discussion}

Oncologic surgery involving the mandible has always represented a reconstructive challenge. From the initial "simple" hemimandibulectomy without reconstruction, to the more complex rehabilitations using microvascular free flaps, the attention to the functional and cosmetic issues has constantly improved..$^{2-4}$ Nevertheless, after the initial enthusiasm and the wide adoption of the microvascular procedures, based on a literature review and the meeting reports, doubts came on about the cost-benefit ratio of free flap reconstruction. Longer surgical sessions, heavier post-operative recovery, huge medical-costs, significant donor site morbidity, and, most of all, an average oncologic outcome worse than expected, all these things promoted a revision of the indications, to be left for younger patients with increased functional needs and better prognostic factors. ${ }^{5-7}$ Myocutaneous pedicle flaps regained interest and indications even though the pectoralis major never stopped to be considered the workhorse in the head and neck reconstruction. ${ }^{8}$ Since the mid-1980s, titanium plaques showed better tolerability and have been adopted for mandibular reconstruction after tumor excision both as a mesh filled with bone chips and as simple plaques embedded in a vascularized tissue..$^{9-11}$

In clinical practice, we encounter cases where a previous multimodality treatment failed but patients' conditions and their
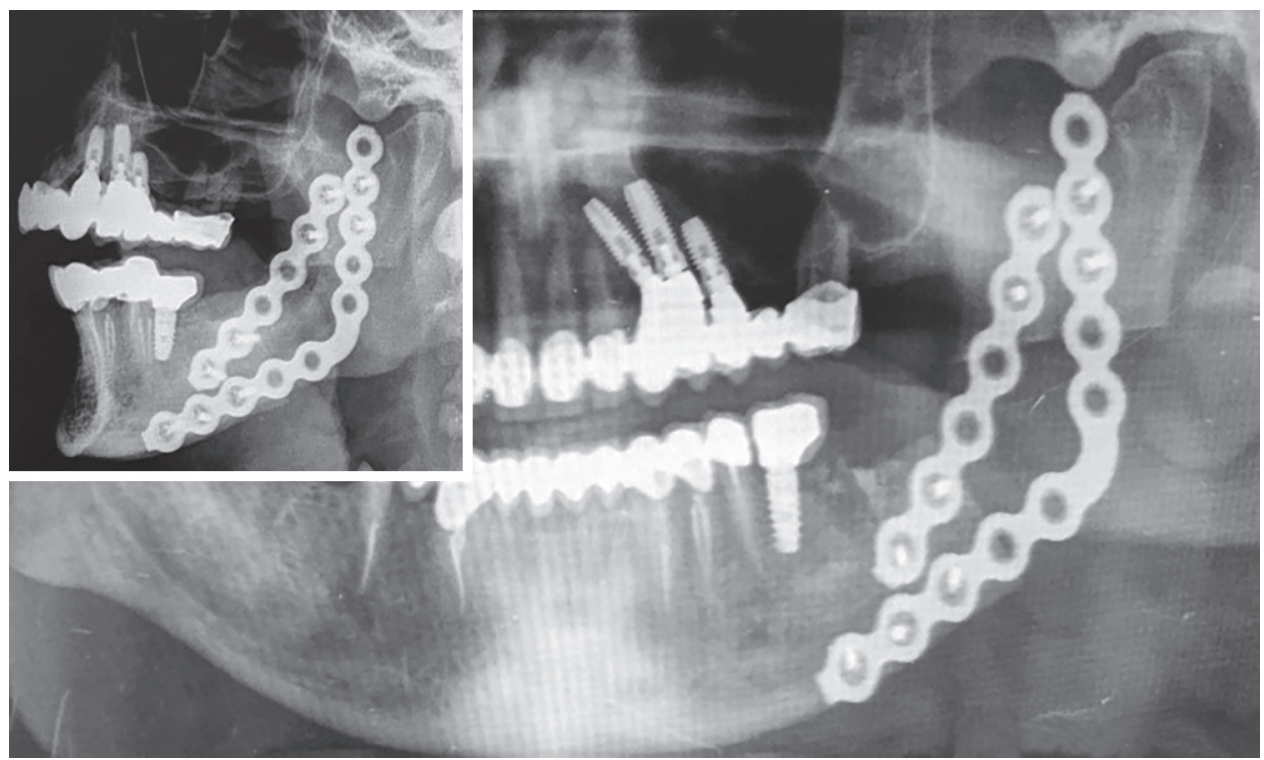

Fig. 1: X-ray control of titanium plate mandibular reconstruction 

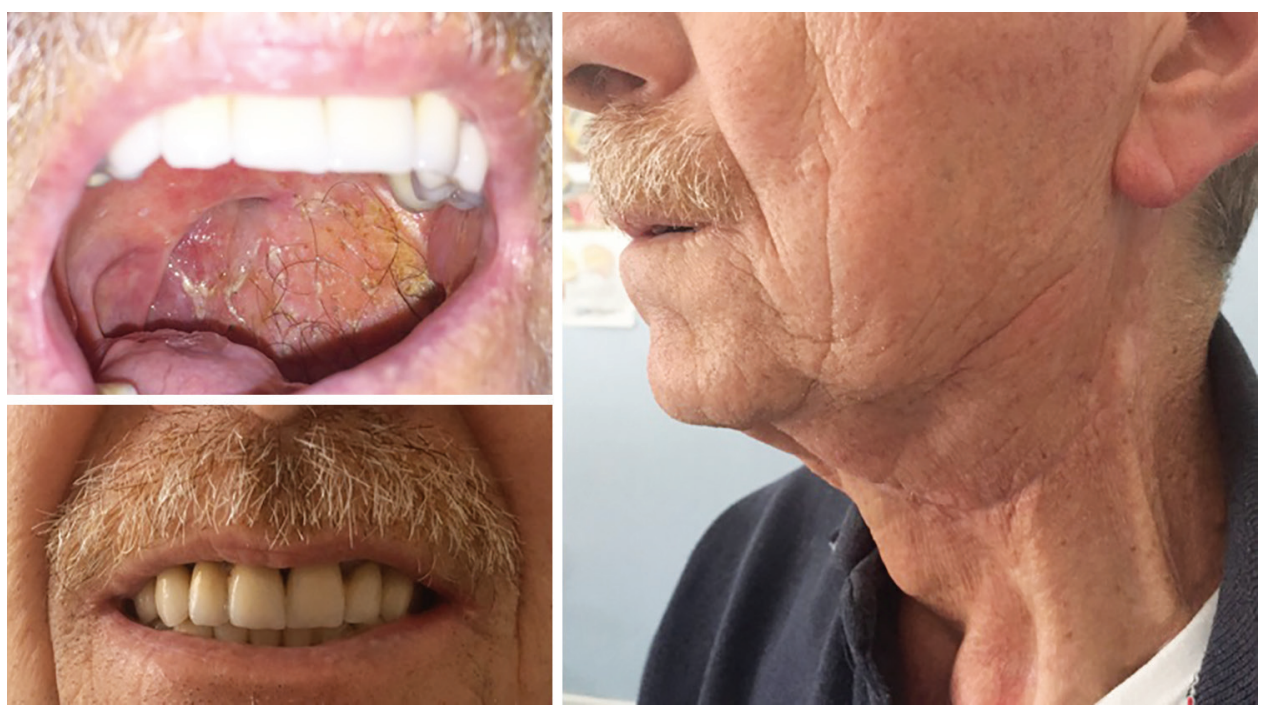

Fig. 2: Appearance of the patient at four-month control (April 2015)

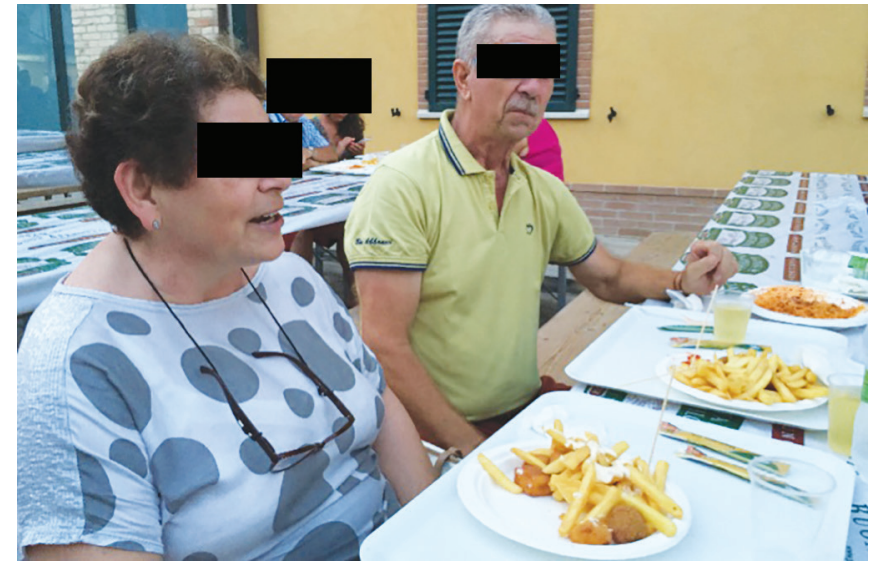

Fig. 3: Patient and his wife at dinner during a village festival (late August 2015)

survival expectation still encourage to undergo a potentially curative program. To such patients we should provide a radical treatment with the shortest hospital stay, least morbidity and more than acceptable cosmetic results.
In our opinion mandibular reconstruction with a titanium plate embedded in a pectoralis major flap is able to satisfy all these requirements.

\section{Conclusion}

Patients with a regrowth of a malignant tumor represent an even more difficult case to take care of. A radical resection with a good prognosis is difficult when impossible to achieve. Pain is the most important issue and its control is mandatory to improve the quality of the patient's residual life. Preservation of an acceptable function and cosmetic result is mandatory too, nowadays more than in the past. When surgery is supposed to be a good option in terms of tumor resection and esthetic functional outcome with a worth residual life expectancy, a safe, quick-recovering, and function-saving technique should be chosen, in order to balance patient's expectations, oncologic prognosis, and organizative costs.

In our experience, mandibular reconstruction with a titanium plate covered with a standard pectoralis major flap demonstrated a good validity and might be considered as the first choice in such cases.
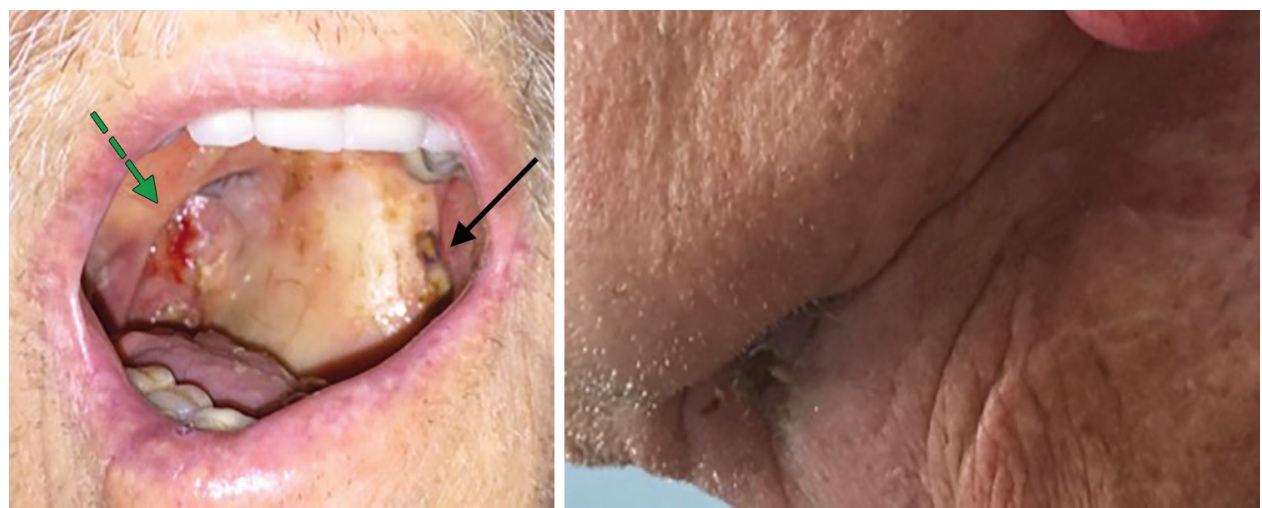

Fig. 4: Patient at (April 2016) control. Left: tumour recurrence, kept under control with palliative chemotherapy (black continuous arrow); bleeding after mucous aspiration, no cancer (green interrupted arrow). Right: mild skin suffering 


\section{References}

1. Dallari S, Zaraca G, et al. Tracheal resection saving the previous supracricoid laryngectomy. Frontiera ORL 2016;VII(2):3-5.

2. Testelin S. History of microsurgical reconstruction of the mandible. Ann Chir Plast Esthet 1992;37(3):241-245.

3. Cordeiro PG, Santamaria E, et al. Mandibular reconstruction. In: Shah JP, Patel SG. ed. Cancer of the Head and Neck. vol. 1. BC Decker Inc; 2001. pp. 358-375.

4. Ferreira JJ, Zagalo CM, et al. Mandible reconstruction: history, state of the art and persistent problems. Prosthet Orthot Int 2015;39(3):182-189.

5. Momeni A, Kattan A, et al. Is microsurgical head and neck reconstruction profitable? Analysis at an academic medical center. Ann Plast Surg 2012;68(4):401-403.

6. Petruzzelli GJ, Brockenbrough JM, et al. The influence of reconstructive modality on cost of care in head and neck oncologic surgery. Arch Otolaryngol Head Neck Surg 2002;128(12):1377-1380.
7. Salvatori $P$, Paradisi $S$, et al. Patients' survival after free flap reconstructive surgery of head and neck squamous cell carcinoma: a retrospective multicentre study. Acta Otorhinolaryngol Ital 2014;34:99-104.

8. Asamura S, Kakizaki H, et al. The Pectoralis Major Myocutaneous Pedicled Flap Revisited. Surg Sci 2013;4:380-384.

9. Salvatori $P$, Motto $E$, et al. Oromandibular reconstruction using titanium plate and pectoralis major myocutaneous flap. Acta Otorhinolaryngol Ital 2007;27(5):227-232.

10. Yamada $\mathrm{H}$, Nakaoka $\mathrm{K}$, et al. Mandibular reconstruction using custom-made titanium mesh tray and particulate cancellous bone and marrow harvested from bilateral posterior ilia. J Plast Surg Hand Surg 2014 Jun;48(3):183-190.

11. Yamashita Y, Yamaguchi Y, et al. Case report. Mandibular reconstruction using a titanium mesh sheet processed by laser welding after segmental mandibulectomy for implant placement. J Oral Maxillofac Surg Med Pathol 2014;26(4):511-514. 